Bundesgesundheitsbl 2009 $\cdot 52: 1011-1018$ DOI 10.1007/s00103-009-0950-1

Online publiziert: 18. September 2009

(c) Springer-Verlag 2009

\author{
H. Kalies ${ }^{1} \cdot$ A. Siedler ${ }^{2}$ \\ ${ }^{1}$ Abteilung Epidemiologie, Institut für Soziale Pädiatrie und \\ Jugendmedizin, Ludwig-Maximilians-Universität, München \\ ${ }^{2}$ Robert Koch-Institut, Berlin
}

\title{
Durchführung von Surveillance- programmen zur Überprüfung der Wirksamkeit von Impfprogrammen am Beispiel von Haemophilus influenzae Typ b und Varizellen
}

\section{Hintergrund und Fragestellung}

Impfungen sind effektive und auch kostengünstige Maßnahmen zur Bekämpfung von Infektionskrankheiten. Ihr Potenzial zur Verringerung von Morbidität und Mortalität hängt zunächst einmal von der Wirksamkeit des Impfstoffes bei der geimpften Person ab. Diese wird normalerweise vor Zulassung eines Impfstoffes in doppeltblinden, randomisierten klinischen Studien untersucht (im Englischen: vaccine efficacy). Die Wirksamkeit eines Impfstoffes nach der Zulassung - also in der Anwendungsrealität (im Englischen: vaccine effectiveness) - kann sich jedoch stark von seiner in klinischen Studien ermittelten Wirksamkeit unterscheiden. Dies kann zum Beispiel der Fall sein, wenn der Impfstoff modifizierter auf den Markt kommt, neue Impfschemata eingeführt oder neue Kombinationen von Impfstoffen verabreicht werden. Außerdem kann in klinischen Studien die langfristige Abnahme der durch den Impfstoff induzierten Immunität nicht untersucht werden, da Studien meist nur ein bis drei Jahre dauern. Schließlich ist die Wirksamkeit eines Impfstoffes im realen Leben noch von weiteren, nicht vollständig standardisierbaren Faktoren wie dem richtigen Transport, seiner Lagerung und Kühlung sowie adäquaten Verabreichung abhängig.

Um die Wirksamkeit eines Impfprogrammes einschätzen zu können, ist nicht nur Wissen über die Wirksamkeit eines Impfstoffes in der Anwendungsre- alität erforderlich, sondern - neben vielen weiteren Aspekten - auch über die Epidemiologie der Krankheit, gegen die geimpft wird. So kann ermittelt werden, ob ein Impfprogramm so weit umgesetzt und akzeptiert wird, dass ein Effekt auf der Populations- (Public-Health-)Ebene sichtbar wird. Hierzu müssen in der Regel hohe Impfquoten erreicht werden (siehe auch Herdenimmunität).

Momentan reichen die in Deutschland verfügbaren Routinedaten nicht aus, um die Wirksamkeit von Impfprogrammen zu evaluieren. Daher müssen zusätzliche Surveillanceprogramme initiiert werden. In welcher Form diese Programme durchzuführen sind, hängt in erster Linie von der Häufigkeit der durch den Impfstoff zu verhindernden Erkrankung ab. Im vorliegenden Beitrag sollen die unterschiedlichen Anforderungen an Surveillanceprogramme für seltene und häufige Erkrankungen herausgearbeitet und anhand je eines Beispiels Möglichkeiten für deren Realisierung dargestellt werden.

\section{Prinzipielle Unterschiede zwischen Surveillanceprogrammen für sel- tene und häufige Erkrankungen}

- Tab. 1 zeigt, wie sich Surveillanceprogramme zur Überprüfung der Wirksamkeit von Impfprogrammen gegen seltene oder häufige Erkrankungen voneinander unterscheiden. Sind die Erkrankungen selten, muss versucht werden, möglichst alle in der Population vorhandenen Er- krankungsfälle zu erfassen. Dies ist sehr wichtig, da hier ein Fall mehr oder weniger das Auswertungsergebnis stärker beeinflusst als bei häufigen Erkrankungen. Da eine Vollerfassung erfolgt, ist die Ermittlung der Erkrankungsinzidenz nach Einführung der Impfung möglich.

Bei Surveillanceprogrammen für häufige Erkrankungen kann unter Umständen ein Populationsbezug fehlen (zum Beispiel Sentinels); dann ist auch die Inzidenz der Erkrankung nicht zu ermitteln. Ist der Impfstatus in der untersuchten Bevölkerung bekannt, kann man die Wirksamkeit des Impfstoffes berechnen [1]; bei häufigen Erkrankungen erlaubt auch die Abnahme der Fallzahlen eine eingeschränkte Aussage über die Wirksamkeit eines Impfprogrammes. In Ausbruchsituationen kann die Impfstoffwirksamkeit jedoch auch nachträglich noch berechnet werden [1].

\section{Das Hib-Surveillancesystem zur Überprüfung der Wirksamkeit hexavalenter Kombinationsimpf- stoffe mit Hib-Komponente als Beispiel für ein Surveillancepro- gramm bei seltenen Erkrankungen}

\section{Hintergrund}

Vor Einführung der Impfung war Haemophilus influenzae Typ b (Hib) der häufigste Erreger von invasiven bakteriellen Infektionen bei Kindern unter fünf Jahren. Nach Einführung der Konjugatimpfstoffe gegen Hib in Deutschland im Jahr 1990 
Tab. 1 Unterschiede zwischen Surveillanceprogrammen zur Überprüfung der Wirksamkeit von Impfprogrammen bei seltenen und häufigen Erkrankungen

\begin{tabular}{|c|c|c|}
\hline & \multicolumn{2}{|c|}{ Surveillanceprogramme zur Überprüfung der Wirksamkeit von Impfprogrammen bei ... } \\
\hline & seltenen Erkrankungen & häufigen Erkrankungen \\
\hline Zu erhebende Population & Alle Fälle & Stichprobe aller Fälle ausreichend \\
\hline $\begin{array}{l}\text { Erhebung des Impfstatus } \\
\text { bei den Fällen }\end{array}$ & $\begin{array}{l}\text { Notwendig, } \\
\text { - um Impfversager zu quantifizieren }\end{array}$ & $\begin{array}{l}\text { Notwendig, } \\
\text { - um Impfversager zu quantifizieren (im Sinne einer } \\
\text { Trendbestimmung) }\end{array}$ \\
\hline $\begin{array}{l}\text { Erhebung des Impfstatus } \\
\text { in der Bevölkerung }\end{array}$ & $\begin{array}{l}\text { Sinnvoll, } \\
\text { - um Akzeptanz und Umsetzung der Impfung zu ermitteln } \\
\text { - um VE zu berechnen }\end{array}$ & $\begin{array}{l}\text { Sinnvoll, } \\
\text { - um Akzeptanz und Umsetzung der Impfung zu ermitteln } \\
\text { - um VE zu berechnen }\end{array}$ \\
\hline $\begin{array}{l}\text { Ermittlung der Inzidenz nach } \\
\text { Einführung der Impfung }\end{array}$ & $\begin{array}{l}\text { Möglich, da alle Fälle in definierter Population erhoben } \\
\text { werden }\end{array}$ & $\begin{array}{l}\text { Nicht ermittelbar, wenn kein Populationsbezug } \\
\text { (Trendaussagen möglich) }\end{array}$ \\
\hline $\begin{array}{l}\text { Berechnung der Wirksamkeit } \\
\text { von Impfstoffen (VE) }\end{array}$ & Möglich, wenn Impfstatus in Population bekannt & (In Ausbruchssituationen punktuell ermittelbar) \\
\hline Beispiel & ESPED-Studie zur Wirksamkeit von Hib-Impfstoffen & Varizellensentinel der AGMV \\
\hline
\end{tabular}

sank die Erkrankungsinzidenz dramatisch [2]. Da für Hib-Impfungen das gleiche Alter galt wie für viele andere empfohlene Impfungen, bestand das Interesse, diese miteinander zu kombinieren. Seit 1996 werden in Deutschland immer höhervalente Kombinationsimpfstoffe verwendet [DTaP-Hib (4-valent), DTaP-Polio-Hib (5-valent)]; Ende 2000 wurde erstmalig ein hexavalenter Kombinationsimpfstoff (DTaP-Polio-IPV-Hib) auf dem deutschen Markt zugelassen. Mit der Zulassung war die Auflage der EMEA an die Impfstoffhersteller verbunden, den Effekt des Impfstoffes auf die Inzidenz invasiver Hib-Erkrankungen bei Kindern zu beobachten und seine Wirksamkeit in der Anwendungsrealität zu ermitteln.

Hintergrund für diese Auflage war, dass in einigen EU-Ländern Bedenken gegen die Verwendung von Hib-Kombinationsimpfstoffen bestanden, da die Hib-Antikörperkonzentrationen nach Grundimmunisierung ohne Booster bei Verwendung von Impfstoffen mit azellulärer Pertussiskomponente geringer waren als bei solchen ohne azellulärer Pertussiskomponente [3]. Die Relevanz dieser Beobachtungen war jedoch umstritten, da die etwas geringeren Hib-Antititer bei Kombinationsimpfstoffen wohl keine Auswirkungen auf ihre Wirksamkeit hatten und sich auch andere Marker für Immunität zwischen den verschiedenen HibImpfstoffen nicht unterschieden [4]. Zudem waren keine Unterschiede mehr festzustellen, wenn eine Booster-Dosis gegeben wurde. Hinzu kam, dass in Großbritannien ab 2002 vermehrt Hib-Impfver- sager gemeldet wurden, deren Auftreten zeitlich mit dem Wechsel von Hib-Kombinationsimpfstoffen mit Ganzkeim-Pertussis-Komponente zu Hib-Kombinationsimpfstoffen mit azellulärer Pertussiskomponente zusammenfiel. Außerdem lag die berechnete Wirksamkeit dieses neuen DTaP-Hib-Kombinationsimpfstoffes nur bei $56,7 \%$ für die Grundimmunisierung ohne Booster $[5,6]$.

Das primäre Ziel des Hib-Surveillance war es, 1. die jährliche Anzahl invasiver Hib-Erkrankungen bei Kindern zu ermitteln und Hib-Impfversager detailliert zu beschreiben, 2. die Inzidenz invasiver Hib-Erkrankungen in Deutschland seit $1998 \mathrm{zu}$ dokumentieren und 3. die Wirksamkeit der hexavalenten Kombinationsimpfstoffe mit Hib-Komponente nach der Zulassung zu bestimmen.

\section{Methoden}

Da invasive Hib-Erkrankungen selten sind, war es wichtig, möglichst alle Erkrankungen bei Kindern unter zehn Jahren in Deutschland zu erfassen. Daher erfolgte die Erhebung über zwei unabhängige Meldesysteme, das heißt über die Erhebungsstelle für seltene pädiatrische Erkrankungen in Deutschland (ESPED) und über ein Labormeldesystem des Robert KochInstituts (RKI). In beiden Surveillancesystemen wurden monatlich Meldekarten an pädiatrische Klinik-Abteilungen (von der ESPED: an zirka 400 verschiedene Abteilungen) beziehungsweise an Labore (vom RKI: an zirka 300 verschiedene Labore) übermittelt, die zu jeweils mehreren aus- gewählten Erkrankungen - darunter Haemophilus influenzae (Hi) - melden sollten. Eine Negativantwort war ebenfalls auf den Meldekarten vorgesehen. Im Falle einer HiMeldung wurde ein detaillierter Fragebogen beziehungsweise ein Interview mit Fragen zu Alter, Geschlecht, Anamnese, Diagnostik inklusive Serotypisierung und HibImpfungen (Anzahl und Impfstoff) versendet/durchgeführt. Bei fehlenden Rückmeldungen wurde mehrmals nachgefragt.

Die Falldefinition für eine invasive Hi-Erkrankung war: Hospitalisation aufgrund einer systemischen Infektion, die klinisch mit einer invasiven Hib-Erkrankung vereinbar war (zum Beispiel Meningitis, Epiglottitis, Cellulitis, Septikämie) mit Isolation des Erregers aus normalerweise sterilen Körperflüssigkeiten wie Blut oder Liquor.

Für die Berechnung der Wirksamkeit eines Impfstoffes müssen detaillierte und zeitnah erhobene Daten über den Impfstatus von repräsentativen Gesunden vorliegen. Routinemäßig werden Daten zum Impfstatus von Kindern bei den Schuleingangsuntersuchungen erhoben. Diese Daten haben jedoch den Nachteil, dass sie vier bis fünf Jahre hinter der aktuellen Entwicklung hinterherhinken (die meisten Impfungen werden für das erste Lebensjahr empfohlen). Zudem werden weder der Zeitpunkt der einzelnen Impfungen noch die Art des Impfstoffes (wichtig zur Beurteilung der Vollständigkeit einer Impfung) erhoben. Um diesen Limitationen zu begegnen, wurde 1998 - in Anlehnung an den jährlich in den USA durchgeführten "national immunisation survey" - ei- 
ne bundesweite repräsentative Telefonumfrage zum Impfstatus von Kindern gestartet. Die Interviews wurden zwischen Juni 1999 und Januar 2008 durchgeführt. Dabei wurden nur Kinder, die zwischen Juli 1996 und Dezember 2005 geboren wurden, berücksichtigt. Insgesamt wurden 94.514 Haushalte kontaktiert und nach der Anwesenheit von Kindern in den oben genannten Geburtskohorten befragt. Lebte ein solches im angesprochenen Haushalt, wurde um ein zweites Interview mit Fragen zu seiner Gesundheit und zum Impfstatus gebeten. In diesem zweiten Interview wurden alle Datumsangaben mit zugehörigen Impfstoffnamen aus dem Impfbuch abgefragt. Zusätzlich wurden das Geburtsdatum und Geschlecht des Kindes und Alter, Wohnort und sozioökonomischer Status der Eltern erfragt.

Mit den Daten aus der Hib-Surveillance bei Kindern und den Impfstatusangaben aus dem Telefonsurvey war die Berechnung der Wirksamkeit der hexavalenten Impfstoffe gegen invasive Hib-Erkrankungen mittels Fall-Kohortenstudie [1] für alle Kinder möglich, die im August 2000 oder später geboren wurden. Dieser Ansatz erlaubt es, den besonders in Deutschland jeweils sehr variablen Zeitpunkt der vier Hib-Impfungen individuell zu berücksichtigen und die Abschätzung der statistischen Varianz (letztlich: Größe des Konfidenzintervalls) korrekt zu berechnen.

\section{Ergebnisse}

In den zwei Erhebungssystemen zu HibErkrankungen lagen die jährlichen Responseraten bei über 90\%, der Anteil der untypisierten Hi-Fälle sank von $25 \%$ im Jahr 1998 auf 19\% im Jahr 2005. Berechnungen zur Vollständigkeit der beiden verwendeten Surveillancesysteme mittels Capture-Recapture-Methoden zeigten, dass das ESPED-Surveillancesystem $36,4 \%$ bis $64,1 \%$ und das Laborerhebungssystem $59,1 \%$ bis $96,6 \%$ aller „wahren" Hi-Fälle pro Jahr in Deutschland erfasste. Zusammen erfassten beide Systeme jedoch zwischen $75,7 \%$ und $98,6 \%$ aller jährlichen Fälle.

Pro Jahr wurden 27 bis 63 Hi-Fälle ermittelt, davon 4 bis 28 Hib-Fälle. $\bullet$ Abb. 1 zeigt alle über die zwei unabhängigen

Bundesgesundheitsbl 2009 · 52:1011-1018 DOI 10.1007/s00103-009-0950-1

(c) Springer-Verlag 2009

H. Kalies · A. Siedler

Durchführung von Surveillanceprogrammen zur Überprüfung der Wirksamkeit von Impfprogrammen am Beispiel von Haemophilus influenzae Typ b und Varizellen

\section{Zusammenfassung}

Um die Wirksamkeit eines Impfprogrammes einschätzen zu können, ist nicht nur Wissen über die Wirksamkeit eines Impfstoffes in der Anwendungsrealität wichtig, sondern - neben vielen weiteren Aspekten - auch über die Epidemiologie der Krankheit, gegen die geimpft wird. So kann ermittelt werden, ob ein Impfprogramm so weit umgesetzt und akzeptiert wird, dass auch ein Effekt auf der Populationsebene sichtbar wird (Herdenimmunität). Momentan sind die in Deutschland verfügbaren Routinedaten nicht ausreichend, um die Wirksamkeit von Impfprogrammen zu evaluieren. Daher müssen zusätzliche Surveillanceprogramme initiiert werden. In welcher Form diese Programme durchgeführt werden müssen, hängt in erster Linie von der Häufigkeit der durch den Impfstoff zu verhindernden Erkrankung ab. Im vorliegenden Beitrag sollen die unterschiedlichen Anforderungen an Surveillanceprogramme für seltene und häufige Erkrankungen herausgear- beitet und anhand je eines Beispiels Möglichkeiten für deren Realisierung dargestellt werden. Als Beispiel für ein Surveillancesystem bei einer seltenen Erkrankung dient die Studie zur Überprüfung der Wirksamkeit hexavalenter Kombinationsimpfstoffe mit Haemophilus-influenzae-Typ-b-Komponente, als Beispiel für ein Surveillancesystem bei einer häufigen Erkrankung dient das Varizellensentinel der Arbeitsgemeinschaft Masern und Varizellen. Diese und andere Surveillancesysteme zur Überprüfung der Wirksamkeit von Impfprogrammen werden nicht oder nur zum Teil durch öffentliche Mittel gefördert. Die möglichen Limitationen anderweitiger finanzieller Förderung werden anhand der genannten Studien diskutiert.

\section{Schlüsselwörter}

Wirksamkeit · Surveillance - Impfprogramm . Varizellen · Haemophilus influenzae Typ b

\section{Surveillance programmes to evaluate the effectiveness of vaccination programmes using vaccination against Haemophilus influenzae type $b$ and varicella as examples}

\section{Abstract}

To evaluate the effectiveness of vaccination programmes, it is not only important to know the effectiveness of the specific vaccine itself but also to have knowledge about the epidemiology of the corresponding vaccinepreventable disease. Only a high acceptance of a vaccination programme by the population will show an effect at the population level (herd immunity). At the moment, data routinely collected in Germany are not sufficient to evaluate the effectiveness of vaccination programmes. Hence, additional surveillance programmes have to be initialised. The frequency of the vaccine-preventable disease in the population under surveillance determines mainly the design of the surveillance. In this article we describe the different requirements for surveillance programmes for common as well as for rare vaccine-preventable diseases. An example for the latter will be the ESPED study on the effectiveness of hexavalent vaccines against Haemophilus influenzae type $b$, an example for the first will be the varicella sentinel of the Working Group on Measles and Varicella. Both surveillance programmes for evaluation of the effectiveness of the respective vaccination programme are financed only partly by the public funds. We discuss the possible limitations of a funding from other sources.

\section{Keywords}

Effectiveness - Surveillance - Vaccination programs · Varicella $\cdot$ Haemophilus influenzae type $b$ 


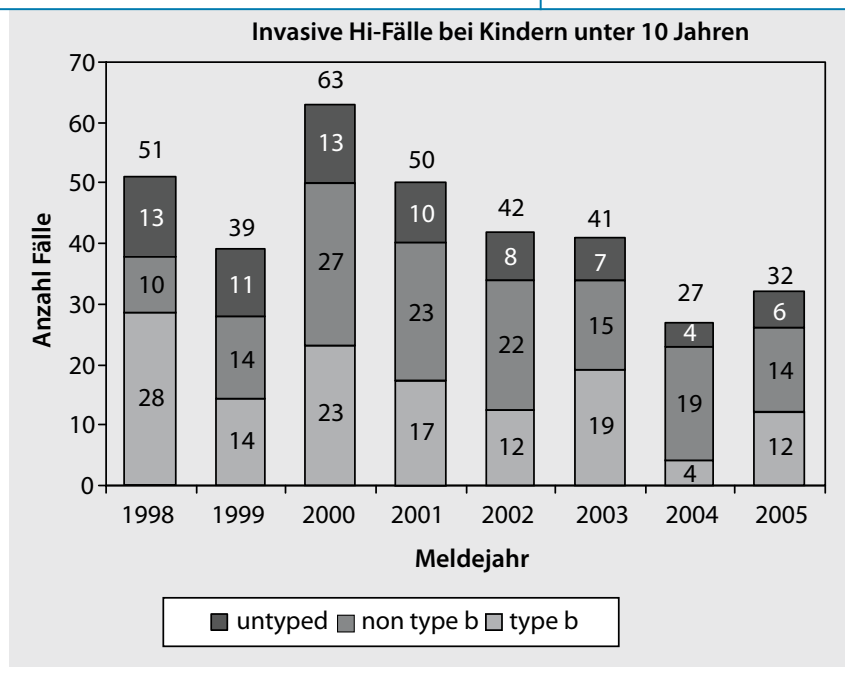

Abb. $1<$ Anzahl invasiver Hi-Fälle bei Kindern unter zehn Jahren nach Serotyp und Jahr (Meldungen aus den zwei Surveillancesystemen: ESPED und Laborerhebung)

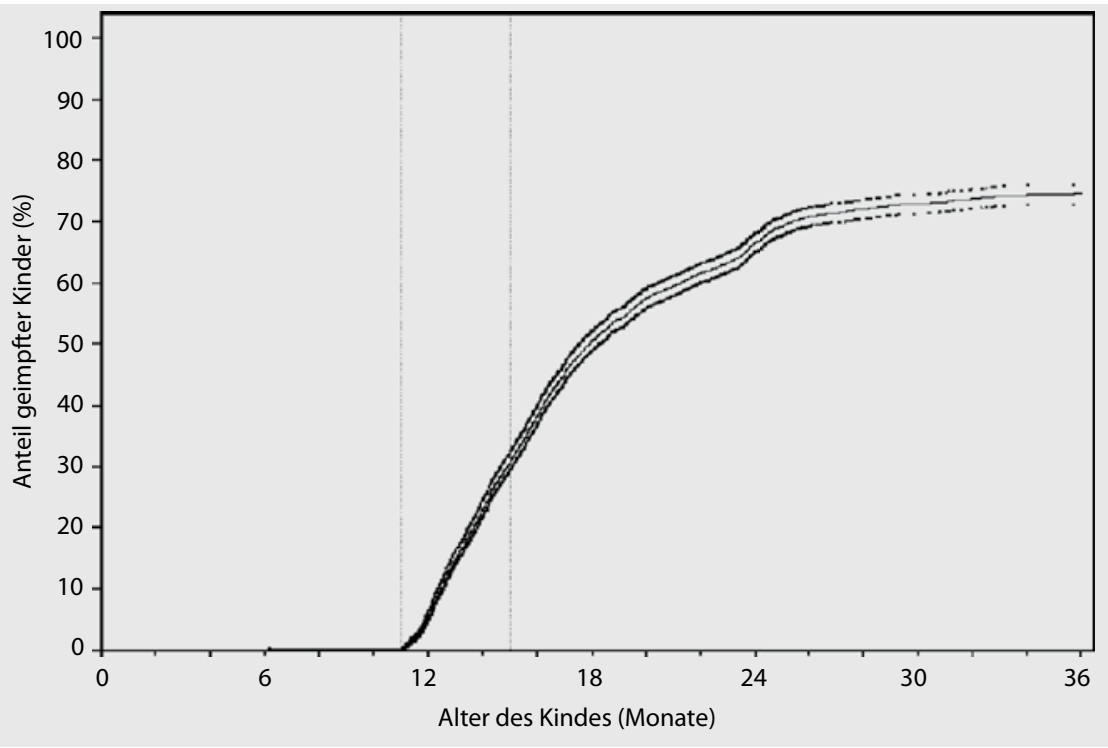

Abb. $2 \Delta$ Anteil der Kinder in Deutschland, die die vollständige Grundimmunisierung mit Booster erhalten haben nach Alter. Inverse Kaplan-Meier-Kurven mit 95\%-Konfidenzintervall. Der von der Ständigen Impfkommission in Deutschland (STIKO) empfohlene Impfzeitraum ist mit vertikalen Linien markiert

Meldesysteme erfassten Fälle pro Jahr und nach Serotyp. Die Inzidenzen lagen für alle invasiven Hi-Erkrankungen zwischen 0,4 und 0,8 pro 100.000 Kinder unter zehn Jahren und Jahr; für Hib-Erkrankungen lagen die Inzidenzen zwischen o,lund o,3, wobei die Gruppe der drei bis elf Monate alten Kinder die höchsten Inzidenzen aufwies. Es gab keinerlei Hinweis auf einen Anstieg invasiver Hi-Infektionen im Beobachtungszeitraum. Diese Zahlen reflektieren sowohl die Wirksamkeit des jeweiligen Impfstoffes als auch eine Herdenimmunität.

Von allen 129 zwischen 1998 und 2005 erfassten invasiven Hib-Fällen wurden 55 mindestens einmal vor Ausbruch der Erkrankung gegen Hib geimpft; 73 wurden nie gegen dagegen geimpft. Von diesen nie geimpften Fällen wären 68, das heißt 93\%, gemäß STIKO-Empfehlung alt genug für zumindest eine Hib-Impfung gewesen. Bei 52 der 55 geimpften Hib-Fälle war das Alter bei Impfung bekannt. Von diesen 52 Kindern erhielten 14 eine inkomplette Grundimmunisierung ohne Booster, 22 eine komplette Grundimmunisierung ohne Booster, zehn eine komplette Grundimmunisierung mit Booster und sechs eine inkomplette Grundimmunisierung mit Booster (zum Beispiel nur eine Impfung im ersten Lebensjahr und eine Booster-Impfung im zweiten Lebensjahr). Wären die Zeitpunkte für eine Impfung gemäß den STIKO-Impfempfehlungen eingehalten worden, hätten 24 der 52 Hib-Fälle (46\%) mindestens eine weitere Impfdosis erhalten können. Insgesamt wurden 15 der 55 geimpften Hib-Fälle mit mindestens einer Dosis eines hexavalenten Impfstoffes geimpft.

Insgesamt wurden 4532 Interviews mit Eltern von Kindern der Geburtskohorten ab 1999 durchgeführt. Die Response-Rate der Telefoninterviews lag bei $66 \%$. Für die Berechnung der Wirksamkeit des hexavalenten Impfstoffes standen 3815 Interviews von Kindern, die nach dem 1. August 2000 geboren wurden, zur Verfügung. Es zeigte sich, dass Kinder sehr zeitverzögert gegen Hib geimpft werden: So hatten zum Beispiel zum Ende des von der STIKO empfohlene Alters von 11,9 Monaten 80,3\% der Kinder eine vollständige Grundimmunisierung ohne Booster und zum Ende des von der STIKO empfohlenen Alters von 17,9 Monaten nur 50,5\% der Kinder eine vollständige Grundimmunisierung mit Booster erhalten. 0 Abb. 2 zeigt diesen Zusammenhang exemplarisch für die vollständige Grundimmunisierung mit Booster. Differenzierte Analysen nach Geburtsjahren seit 1999 konnten zeigen, dass mit der Einführung immer höhervalenter Impfstoffe auch diese Zeitverzögerung abnimmt [7].

Die Berechnung der Wirksamkeit hexavalenter Kombinationsimpfstoffe mit Hib-Komponente zeigte hohe Werte für die vollständige Grundimmunisierung und die Boosterimpfung (• Tab. 2). So liegt zum Beispiel die Wirksamkeit für die vollständige Grundimmunisierung ohne Booster bei 90,4\% (95\%-Konfidenzintervall: $70,6-96,8)[8]$.

\section{Schlussfolgerung}

Fünf Jahre nach Einführung hexavalenter Kombinationsimpfstoffe mit Hib-Komponente in Deutschland lassen sich keinerlei Hinweise darauf finden, dass sich die Fallzahlen beziehungsweise Inzidenzen für invasive Hib-Erkrankungen bei Kindern erhöht haben. Die Hälfte aller ermittelten Impfdurchbrüche hätte wahrscheinlich vermieden werden können, wenn die Kinder zeitgerecht geimpft worden wären. Hib-Impfungen werden zunehmend akzeptiert und immer zeitgerechter umgesetzt. Diese Beobachtung scheint auch im Zusammenhang mit der Einführung immer höhervalenter Impfstoffe zu stehen, 
Tab. 2 Wirksamkeit der hexavalenten Impfstoffe gegen Haemophilus influenzae Typ b bei Kindern, die zwischen August 2000 und Dezember 2004 in Deutschland geboren wurden [8]. Schätzer, Standardfehler und Impfstoffwirksamkeit wurden mittels Cox-Regression mit zeitabhängigen Kovariablen und robusten Varianzschätzern ermittelt

\begin{tabular}{|llllll}
\hline Impfschema & Anzahl Fälle & Schätzer ( $\beta)$ & Standardfehler & Impfstoffwirksamkeit & 95\%-Konfidenzintervall \\
Keine Impfung & 19 & Referenz & - & Referenz & - \\
$\begin{array}{l}\text { Unvollständige Grundimmunisierung (GI) } \\
\text { ohne Booster }\end{array}$ & 6 & $-1,1511$ & 0,4795 & $68,4 \%$ & $19,0-87,6$ \\
Vollständige Gl ohne Booster & 5 & $-2,3402$ & 0,5698 & $90,4 \%$ & $70,6-96,8$ \\
Vollständige Gl mit Booster & 0 & $-16,7399$ & 0,4273 & $100,0 \%$ & $52,7-100,0$
\end{tabular}

sodass für die Verabreichung der gleichen oder sogar größeren Anzahl von Impfantigenen weniger Arztkonsultationen erforderlich sind. Die Wirksamkeit der hexavalenten Kombinationsimpfstoffe mit Hib-Komponente ist gut und mit der von vier oder fünfvalenten Hib-Impfstoffen vergleichbar [9]. Capture-Recapture-Berechnungen haben gezeigt, dass aber auch durch die zwei unabhängigen aktiven Surveillance-Systeme nicht alle „wirklich vorhandenen“ Fälle ermittelt werden konnten. Diese Methode erlaubt es jedoch, diese Unterschätzung zu quantifizieren.

\section{Das Varizellensentinel der Arbeits- gemeinschaft Masern und Varizel- len als Beispiel für ein Surveillan- ceprogramm zur Überprüfung der Wirksamkeit von Impfprogram- men bei häufigen Erkrankungen}

\section{Hintergrund}

Im Juli 2004 hat die STIKO die Varizellenimpfung für alle Kinder im Alter von elf bis 14 Monaten sowie für ungeimpfte Kinder und Jugendliche ohne Varizellenanamnese empfohlen [10]. Mit der generellen Impfempfehlung wird das Ziel verfolgt, die Morbidität durch Varizellen in Deutschland und insbesondere damit verbundene Komplikationen, Hospitalisierungen und Todesfälle signifikant zu reduzieren.

Varizellenerkrankungen unterliegen nicht der Meldepflicht, einzige Routinedatenquellen sind die Hospital- und Todesursachenstatistik, die aber keinen Rückschluss auf die aktuelle epidemiologische Situation zulassen, da diese Daten nur mit zeitlicher Verzögerung vorliegen und außerdem nur einen begrenzten Ausschnitt der Morbidität wiedergeben. Daher wurde im April 2005 die bereits seit 1999 bestehende Arbeitsgemein- schaft Masern zur Arbeitsgemeinschaft Masern und Varizellen (AGMV) erweitert und dem Masernsentinel ein Sentinelsystem Varizellen hinzugefügt.

Das primäre Ziel des Sentinels ist es, Trends in der Häufigkeit der Varizellenerkrankungen und bei ihren Komplikationen in allen Altersgruppen festzustellen. Daneben sollen die Häufigkeit von Erkrankungen nach Varizellenimpfung, die Anzahl von Herpes-Zoster-Erkrankungen sowie die Häufigkeit von Impfungen im Sentinel erhoben werden.

\section{Methoden}

Von mehr als 1100 niedergelassenen Ärzten werden seit April 2005 ehrenamtlich und mit großem Meldeaufwand Daten zu Varizellenerkrankungen zur Verfügung gestellt $[11,12,13,14]$. Monatlich werden mit standardisierten Fragebögen aggregierte Angaben zu Varizellenerkrankungen in vorgegebenen Altersgruppen, die Zahl der behandelten Patienten mit den gesuchten und definierten Erkrankungsbildern (Komplikationen der Varizellen, Varizellen bei Geimpften, Herpes Zoster bei geimpften und ungeimpften Personen), die Impfzahlen für Masern und Varizellen (jeweils unterschieden nach erster und zweiter Dosis), die verabreichten Kombinations- oder monovalenten Varizellenimpfstoffe sowie die Gesamtzahl der Patientenkontakte in allen angemeldeten Praxen abgefragt. Wurden keine Patienten mit Varizellen behandelt, ist eine Nullmeldung erwünscht. Fehlende Monatsmeldungen werden nachgefragt. Darüber hinaus ist für definierte Einzelfälle (Komplikationen der Varizellenerkrankung, Herpes Zoster, Varizellen bei Geimpften und Varizellen bei Erwachsenen) ein weiterer Fragebogen mit zusätzlichen klinischen und anamnestischen Angaben zum Einzelfall auszufüllen. Auf allen Fragebögen sind Falldefinitionen für die abgefragten Kategorien enthalten. Die Bezugsbasis für die jeweiligen Meldezahlen sind die Gesamtzahl der Praxiskontakte pro Monat beziehungsweise die Zahl der Meldepraxen.

\section{Erste Ergebnisse}

Die angemeldeten Sentinelärzte sind in allen Bundesländern in etwa der gleichen Relation verteilt wie die Gesamtzahl der Pädiater und Nichtpädiater, das heißt zirka 15\% aller Pädiater und o,8\% aller Allgemeinmediziner sind im Sentinel angemeldet. Monatlich beteiligen sich zwischen 400 und 520 Pädiater und 160 bis 275 Allgemeinmediziner und hausärztlich tätige Internisten. Aufgrund der Größe und Struktur des Ärztekollektivs und der Tatsache, dass Praxen aller Größen aus unterschiedlich großen ländlichen und städtischen Gebieten vertreten sind und die Meldebeteiligung relativ konstant ist, erfüllt das Sentinel die Anforderungen, die an eine bundesweite repräsentative Stichprobe gestellt werden.

Von April 2005 bis Dezember 2008 wurden von 1177 Ärzten insgesamt 79.625 Varizellenerkrankungen gemeldet. Die Zahl der gemeldeten Varizellenfälle zeigte jahreszeitliche Schwankungen - die wenigsten Fälle gab es jeweils von August bis Oktober, die meisten von März bis Mai. Der Schwerpunkt der gemeldeten Varizellenerkrankungen lag bei den unter fünfjährigen Kindern, wobei ihr Anteil von 65\% im ersten Jahr auf $53 \%$ der Fälle in der laufenden Saison zurückgegangen ist. Von 2005 bis 2008 waren die im Sentinel gemeldeten Varizellenerkrankungen in allen Altersgruppen rückläufig (• Abb. $\mathbf{3})$. Der stärkste Rückgang war bei den ein- bis zweijährigen Kindern zu beobachten. Bei ihnen wurden etwa $60 \%$ weniger Fälle pro Praxis in der dritten im Vergleich zur ersten Saison gemeldet. Der rückläufige Trend der Varizellenfälle setzte sich auch in der vierten 


\section{Leitthema}

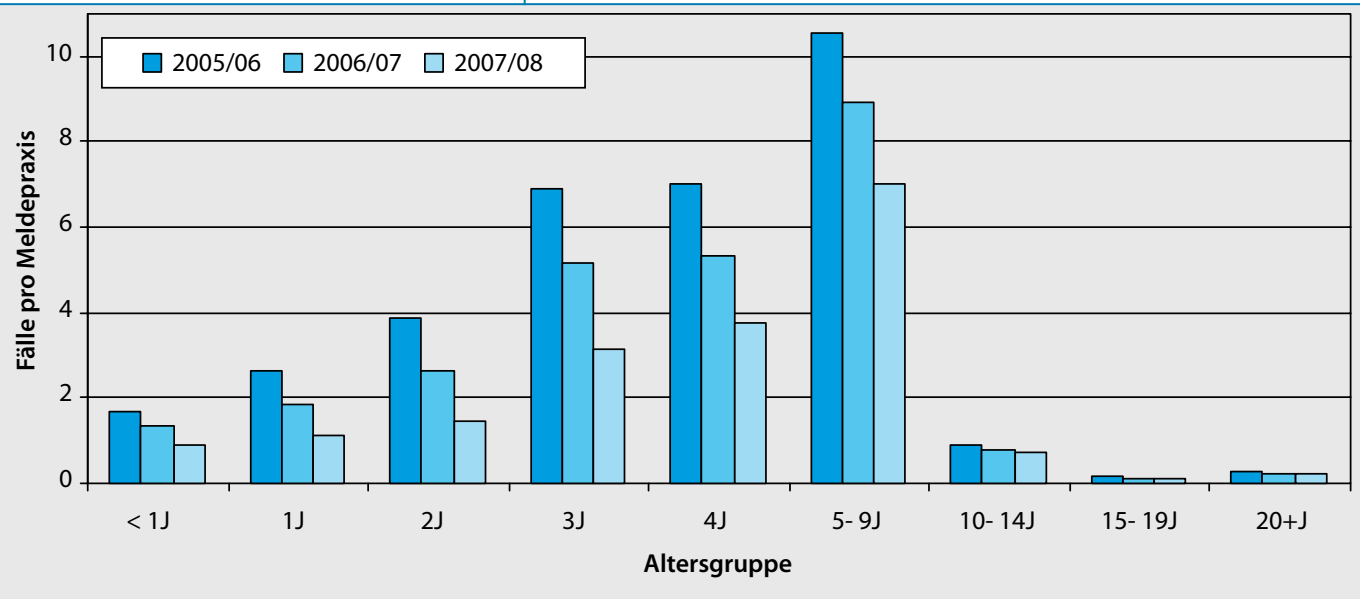

Abb. $3<$ Anzahl der Varizellenfälle nach Altersgruppen pro Meldepraxis in drei aufeinanderfolgenden Saisons, Varizellensentinel der Arbeitsgemeinschaft Masern und Varizellen (AGMV)

Saison fort: Von April bis Dezember 2008 wurden in fast jedem Monat pro Meldepraxis weniger Varizellenfälle registriert als im selben Monat des Vorjahres; im September waren es etwa genauso viele. Des Weiteren wurden insgesamt 306 Komplikationen bei Varizellenerkrankung (etwa 0,4\% der Erkrankten) gemeldet, in 276 Fällen liegen Einzelfallbeschreibungen der aufgetretenen Komplikationen vor. Die häufigsten Nennungen seien hier aufgezählt: 141-mal kam es zu Komplikationen der Haut (davon 105-mal zu einer bakteriellen Superinfektion der Haut), 47 Patienten litten an einer Otitis media, 21 an einer Pneumonie, je sechsmal kam es zu Enzephalitis und Zerebellitis.

Insgesamt wurden 5816 Fälle von Herpes Zoster aufgeführt, von denen 4013 im Einzelnen beschrieben wurden. Danach liegt der Erkrankungsschwerpunkt bei den älteren Erwachsenen. Bisher sind keine Verschiebungen in den Altersgruppen zu beobachten.

Varizellen bei Geimpften kamen 2270mal zur Meldung, ihr Anteil an allen Varizellenerkrankungen erhöhte sich von $1 \%$ in der ersten auf $8 \%$ in der laufenden Saison. Für 1907 Patienten liegen Einzelfallbeschreibungen vor: Von ihnen erkrankten 258 (6) vor Ablauf von 42 Tagen nach der ersten (zweiten) Impfung an Varizellen (mittlerer Abstand elf Tage). Der Altersmedian bei Impfung lag bei vier Jahren. Bei 44 Patienten wurden virologische Untersuchungen veranlasst. Dabei wurde in 29 Fällen Wildvirus nachgewiesen, das heißt, dass die Patienten zum Zeitpunkt der Impfung bereits infiziert waren, viermal wurde Vakzine-Virus und damit sogenannte Impfvarizellen nachgewiesen, elfmal wurde kein Virus gefunden. Ei- nen zeitlichen Abstand zur Impfung von mehr als 42 Tagen wiesen 1643 geimpfte Personen mit Varizellen auf, das heißt, es handelte sich um Durchbruchserkrankungen. Der mittlere Abstand zur Impfung lag bei 23 Monaten, der Altersmedian zum Zeitpunkt der Impfung bei zwei Jahren. In 226 von 288 im HerpesvirenLabor des RKI untersuchten Proben von Patienten mit Durchbruchserkrankung wurde Wildvirus nachgewiesen.

Monatlich wurden von den Sentinelärzten der AGMV auch die durchgeführten Varizellen- und Masern-Impfungen gemeldet. Es wurde deutlich, dass die Umsetzung der Varizellenimpfempfehlung der STIKO von der Kostenübernahme durch die Krankenkassen abhängig war. Die Zahl der Impfungen nahm zunächst - regional unterschiedlich und eng an die Kostenerstattung gekoppelt - zu und verdoppelte sich insgesamt von weniger als sechs Impfungen pro Praxis im April 2005 auf 12 im Sommer 2006. Im Jahr 2008 lagen die Zahlen der durchgeführten ersten Masernund Varizellenimpfungen auf stabilem $\mathrm{Ni}$ veau, und es wurden jeweils zwischen acht und zehn Impfungen pro Meldepraxis und Monat durchgeführt. Mit der Verfügbarkeit des MMRV-Kombinationsimpfstoffes (MMRV: Masern, Mumps, Röteln, Varizellen) stieg seine Verwendung bei den Sentinelärzten stark an. Im Jahr 2008 waren bereits 70\% aller Varizellendosen im Sentinel mit Kombinationsimpfstoff verimpft worden. Wegen der Zwei-Dosen-Zulassung dieses Impfstoffes stieg insbesondere auch die Zahl der zweiten Impfdosen für Varizellen stark an.

Das Verhältnis der Varizellenimpfungen (erste Dosis) zu den altersgleich empfohlenen ersten Masernimpfungen kann als Marker für die Umsetzung der Impfempfehlung angesehen werden. Während zu Beginn der Sentinelerhebung verglichen mit ersten Varizellenimpfungen noch etwa doppelt so viele erste Masernimpfungen verabreicht wurden, werden seit Herbst 2006 pro Meldearzt und Monat gleich viele erste Varizellen- und erste Masernimpfungen durchgeführt.

\section{Schlussfolgerung}

Insgesamt gesehen, arbeitet das Meldesystem des Varizellensentinels der AGMV stabil und erlaubt es, Trends der Morbiditätsentwicklung zu schätzen. Selbst wenn man säkuläre Schwankungen bei der Varizellenmorbidität in Betracht ziehen würde - für die es wegen der fehlenden Varizellensurveillance vor der allgemeinen Impfempfehlung aber leider keine Hinweise geben kann -, zeigen der Rückgang der Fallzahlen in vier aufeinanderfolgenden Saisons sowie der altersspezifische Rückgang der Varizellenfälle einen rückläufigen Trend der Varizellenmorbidität. Dies deutet auf eine erfolgreiche Umsetzung des Impfprogramms hin. Die labordiagnostische Absicherung ermöglicht eine differenzierte Erfassung von Durchbruchserkrankungen. Über die angegebene Anzahl der durchgeführten Impfungen (erste beziehungsweise zweite Impfdosis Varizellen, MMRV und Masern) lässt sich der Trend bei der Umsetzung und Akzeptanz der Impfempfehlung abbilden.

Eine Hochrechnung auf die Varizellenmorbidität in Deutschland ist auf Basis der Sentineldaten prinzipiell nicht möglich, da der Bevölkerungsbezug als Nenner fehlt. Eine bevölkerungsbezogene Erhebung ist aber zur Beobachtung alter- 
spezifischer Inzidenzen notwendig. Diese lässt sich auch stichprobenartig (zum Beispiel als Sentinel) realisieren, wenn eine Gesamterhebung in einer definierten Region gelingt.

\section{Aufgabenverteilung und finanzielle Förderung}

Die beschriebenen Surveillanceprogramme zur Überprüfung der Wirksamkeit von Impfprogrammen werden nur teilweise durch Staatsmittel gefördert, ein großer Anteil wird von pharmazeutischen Unternehmen finanziert.

Die AGMV ist eine gemeinsame Initiative des RKI sowie der Impfstoffhersteller GlaxoSmithKline $\mathrm{GmbH}$ und Sanofi Pasteur MSD unter Beteiligung des Deutschen Grünen Kreuzes (DGK). Das RKI hat die wissenschaftliche Federführung. Es wird dabei von einem Wissenschaftlichen Beirat unterstützt. Dem Beirat gehören externe Experten der Epidemiologie, Kinderheilkunde, Allgemeinmedizin, Virologie sowie des öffentlichen Gesundheitsdienstes an. Neben den Datenanalysen werden im RKI außerdem die Laboruntersuchungen durchgeführt. Das DGK ist verantwortlich für die Teilnehmerrekrutierung, den Kontakt zu den teilnehmenden Ärzten, das gesamte Datenmanagement sowie den Internetauftritt (http://www.agmv.de). Es hat dafür eine Agentur beauftragt, deren Arbeit von den Impfstoffherstellern finanziert wird. Das RKI erhält keine finanziellen Zuwendungen von den Impfstoffherstellern. Die strukturellen und finanziellen Gegebenheiten lassen derzeit jedoch wenig Spielraum für die inhaltliche Gestaltung und Weiterentwicklung des bisher genutzten Erhebungsinstrumentes. Reibungs- und Zeitverluste bei der Bereitstellung von Daten durch Dritte sind nicht immer zu vermeiden, gehen jedoch zulasten der Datenqualität und können zeitnahe und stratifizierte Auswertungen beeinträchtigen. Entscheidungen über den Ablauf und Inhalt der Erhebung können nur im Konsens aller AGMV-Kooperationspartner getroffen werden. Die Finanzierung wird jeweils in Zweijahresschritten geregelt. Als finanzielle Förderer versuchen die Hersteller auch auf die inhaltliche Gestaltung Einfluss zu nehmen.
Die Hib-ESPED-Studie resultierte aus Auflagen der EMEA an die Impfstoffhersteller GlaxoSmithKline GmbH und Sanofi Pasteur MSD. Sie verfügten jedoch über keinerlei Logistik, um eine solche Studie durchzuführen. Die Auftraggeber und damit Finanzierer der Hib-ESPEDStudie waren die beiden Impfstoffhersteller. Die Hib-ESPED-Studie wurde federführend am Institut für Soziale Pädiatrie (München) durchgeführt. Die ESPEDKarten und -Fragebögen wurden über die ESPED-Zentrale in Düsseldorf an die Kliniken verschickt und von dieser dort auch wieder nachgefragt. Mit der Durchführung des nationalen Telefonsurvey zur Erhebung des Impfstatus wurde eine Firma beauftragt. Das Labormeldesystem wurde vom RKI geplant, durchgeführt und auch finanziert. Für zusätzliche Befragungen zu den Labormeldungen über invasive HiInfektionen standen für Personal externe Drittmittel von der Stiftung „Präventive Pädiatrie“ in Mainz zur Verfügung. Alle Daten flossen am Institut für Soziale Pädiatrie zur Auswertung zusammen.

Für invasive Erkrankungen durch Haemophilus influenzae besteht außerdem eine Meldepflicht nach $₫ 7$ Infektionsschutzgesetz (IfSG) durch das diagnostizierende Labor bei direktem Erregernachweis aus Blut oder Liquor. Ein Abgleich der Meldungen aus den beiden beschriebenen Studienarmen mit den invasiven Hi-Fällen bei Kindern, die nach IfSG in den Jahren 2001 bis 2005 von den Gesundheitsämtern übermittelt wurden, zeigte, dass sich durch ein passives Surveillancesystem wie der Meldepflicht die Epidemiologie seltener Erkrankungen nur sehr unvollständig abbilden lässt: Weniger als die Hälfte der in allen drei Systemen (das heißt ESPED, Labor, IfSG) gemeldeten Fälle fand sich auch in den IfSG-Meldungen wieder [15]. Trends in der Entwicklung von Hib-Fällen und bei Durchbruchserkrankungen bei Kindern konnten nach IfSG aufgrund der geringen Typisierungsraten und der unvollständigen Angaben zum Impfstatus gar nicht verfolgt werden $[15,16]$.

Die vielfach geforderte Meldepflicht für alle impfpräventablen Erkrankungen könnte zwar wichtige epidemiologische Basiszahlen liefern. Sie ist aber unter Aspekten der Häufigkeit der Zielerkrankung, ihrer Erfassbarkeit und Diagnosesicherheit sowie nicht zuletzt wegen der Möglichkeit und Notwendigkeit von Interventionen durch den öffentlichen Gesundheitsdienst differenziert zu betrachten und nicht immer das Mittel der Wahl. Zur Überprüfung der Wirksamkeit von Impfprogrammen sind daher in Ergänzung zur Meldepflicht weitere Surveillancesysteme notwendig. Zur dauerhaften Sicherung dieser Surveillance ist angesichts des hohen Stellenwertes sowie der Public-Health-Relevanz der resultierenden Daten eine industrieunabhängige Finanzierung dringend angezeigt. Nur so kann die Öffentlichkeit von der Objektivität der Ergebnisse überzeugt werden. Andernfalls bleiben Glaubwürdigkeitsprobleme, die auch der Förderung des Impfgedankens abträglich sind. Bei industrieabhängigen Finanzierungen besteht die Gefahr, dass Planung und Zielsetzung der Studien inhaltlich beeinflusst werden. Hinzu kommt, dass bei möglichen Problemen mit dem jeweiligen Impfstoff leicht Interessenskonflikte zwischen öffentlichem und Industrieinteresse entstehen können, die zumindest eine sehr sorgfältige Berücksichtigung in den jeweiligen Studienprotokollen finden müssen (Interpretationshoheit der Daten, Veröffentlichungsrechte).

In diesem Sinne ist die auf der 1. Nationalen Impfkonferenz im März 2009 in Mainz getroffene Forderung zu begrüßen, Versorgungsforschung, wie zum Beispiel Nachzulassungsstudien, zur Wirksamkeit allgemeiner Impfempfehlungen als öffentliche Aufgabe zu betrachten und daher verstärkt zu fördern und durchzuführen [17]. Es bleibt zu ergänzen, dass hier ein Gesamtkonzept wünschenswert wäre, das Strukturen schafft und die begrenzt vorhandenen Ressourcen bündelt, um inhaltliche und organisatorische Fragen künftiger Sentinels oder anderer Erhebungssysteme, die Entscheidungsgrundlagen für Impfstrategien und damit für wesentliche gesundheitspolitische Entscheidungen liefern sollen, nachhaltig zu regeln.

\section{Korrespondenzadresse \\ Dr. H. Kalies}

Abteilung Epidemiologie, Institut für Soziale

Pädiatrie und Jugendmedizin,

Ludwig-Maximilians-Universität

Heiglhofstr. 63, 81377 München

helen.kalies@med.uni-muenchen.de 


\section{Literatur}

1. Kalies H, von Kries R (2006) Messung der Wirksamkeit von Impfstoffen. In: Schlipköter U, Wildner M (Hrsg) Lehrbuch Infektionskrankheiten. Huber, Bern

2. von Kries R, Heinrich B, Böhm O et al (1997) Systemic Haemophilus influenzae disease in Germany: 1992-1995. Monatsschr Kinderheilkd 145:136143 (in German)

3. Eskola J, Olander RM, Hovi T et al (1996) Randomised trial of the effect of co-administration with acellular pertussis DTP vaccine on immunogenicity of Haemophilus influenzae type b conjugate vaccine. Lancet 348:1688-1692

4. Eskola J, Ward J, Dagan R et al (1999) Combined vaccination of Haemophilus influenzae type $b$ conjugate and diphtheria-tetanus-pertussis containing acellular pertussis. Lancet 354:2063-2068

5. Trotter CL, Ramsey ME, Slack MPE (2003) Rising incidences of Haemophilus influenzae type $b$ disease in England and Wales indicates a need for second catch-up vaccination campaign. Comm Dis Pub Health 6:55-58

6. Ramsey ME, McVernon J, Andrews NJ et al (2003) Estimating Haemophilus influenzae type $b$ vaccine effectiveness in England and Wales by use of the screening method. J Infect Dis 188:481-485

7. Kalies H, Grote V, Verstraeten T et al (2006) The use of combination vaccines has improved timeliness of vaccinations in children. Pediatr Infect Dis J 25(6):507-512

8. Kalies H, Grote V, Siedler A et al (2008) Effectiveness of hexavalent vaccines against invasive Haemophilus influenzae type b disease: Germany's experience after 5 years of licensure. Vaccine 26(20):2545-2552

9. Kalies H, Verstraeten T, Grote V et al (2004) 4 1/2 year follow-up of the effectiveness of DTaP/Hib and DTaP-IPV/Hib combination vaccines in Germany. Pediatr Infect Dis J 23:944-950

10. Empfehlungen der Ständigen Impfkommission (STIKO) am Robert Koch-Institut (2004) Stand: Juli 2004. Epid Bull 30:235-250

11. RKI (2005) AGM/V: Masern-Sentinel (AGM) wird durch Sentinel der Varizellen ergänzt. Epid Bull 13:111-112

12. RKI (2005) Varizellen-Sentinel der AGM/V erfolgreich gestartet: Erste Ergebnisse des Datenrücklaufs und Erkenntnisse zur Umsetzung der Varizellen-Impfung. Epid Bull 36:329-332

13. RKI (2006) Zur Sentinel-Surveillance der Varizellen in Deutschland. Erfahrungen nach Ablauf des ersten Jahres. Epid Bull 36:307-309

14. RKI (2008) Zur Sentinel-Surveillance der Varizellen in Deutschland. Bericht aus dem Zeitraum April 2005 bis November 2007. Epid Bull 8:61-64

15. Milde-Busch A, Kalies $H$, Ruckinger $S$ et al (2008) Surveillance for rare infectious diseases: is one passive data source enough for Haemophilus influenzae? Eur J PubH 18:371-375

16. RKI (2008) Infektionsepidemiologisches Jahrbuch meldepflichtiger Krankheiten für 2007. Eigenverlag, Berlin, Kapitel 6.15 Haemophilus influenzae, invasive Erkrankung 84-87

17. Pressemitteilung 7.3.2009: 1. Nationale Impfkonferenz schafft Rahmen für einen „Nationalen Impfplan“ aller Bundesländer. Herausgeber: Ministerium für Arbeit, Soziales, Gesundheit, Familie und Frauen Rheinland-Pfalz

\section{Neuer Ansatz gegen Krebs: die Immunsuppression des Tumors aushebeln}

Krebs entsteht durch unkontrolliertes Wachstum von normalen Körperzellen. Im Prinzip kann das Immunsystem das Wachstum von Tumorzellen erkennen und sie über natürliche Killerzellen und zytotoxische T-Lymphozyten direkt zerstören. Um den Angriff des körpereigenen Immunsystems abzuwehren, sezernieren Tumoren immunsuppressive Moleküle, die die Immunantwort dämpfen und Zellen anlocken, die ihrerseits ebenfalls immunsuppressiv wirken. Sie heißen regulatorische T-Zellen ("Treg"-Zellen) und haben normalerweise die Funktion, Immunreaktionen gegen körpereigenes Gewebe zu unterbinden, die sonst zu Autoimmunerkrankungen führen würden. Zusammen mit den Treg-Zellen erschweren die von den Tumoren gebildeten immunsuppressiven Moleküle es dem Immunsystem, den wachsenden Tumor anzugreifen. In der Folge kommt es zu unkontrolliertem Wachstum und Krebserkrankung.

Alternative Ansätze zur Krebstherapie basieren auf der gezielten Stimulation des Immunsystems, um den Tumor auszuschalten. Unter diesem Aspekt wurden bereits zahlreiche biologische Therapien entwickelt. Einige davon haben die Aktivierung dendritischer Zellen zum Ziel. Diese besonderen Zellen des Immunsystems sind in der Lage (Tumor-)Antigene so zu präsentieren, dass die T-Lymphozyten zur Abtötung der Tumorzellen befähigt werden. Nur wenige dieser Ansätze haben sich als wirksam gegenüber einzelnen Tumorarten erwiesen. Ein Problem der TLRAgonisten besteht darin, dass sie sowohl immunsuppressive als auch entzündliche Immunantworten anstoßen können, so dass sie die T-Zellen in verschiedene Richtungen - entweder tumoraggressiv oder immunsuppressiv beeinflussen können. Diese Eigenschaft soll den Kollateralschaden während einer Infektion oder Entzündung begrenzen, schränkt aber die Fähigkeit der TLR-Agonisten ein, im immunsupprimierten Umfeld eines
Tumors eine wirksame antitumorale T-ZellAntwort auszulösen.

Wissenschaftlern des Trinity College Dublin gelang es jedoch, die durch TLR-Agonisten ausgelöste Immunantwort so zu manipulieren, dass der immunsuppressive Arm gezielt ausgeschaltet wird. Die Wissenschaftler erreichten dies durch Manipulation der Signalwege innerhalb der dendritischen Zellen, durch die die Induktion der immunsuppressiv wirkenden von Treg-Zellen unterbunden wird. Damit wird der alternative Weg zu einer schützenden Immunantwort freigemacht. Dieser neue Ansatz war in Tumormodellen an der Maus bereits sehr erfolgreich und könnte auch beim Menschen immuntherapeutische Therapien gegen Krebs wirksam verstärken.

Quelle:

Deutsche Gesellschaft für Immunologie, www.immunologie.de 